\title{
miR-628-3p regulates osteoblast differentiation by targeting RUNX2: Possible role in atrophic non-union
}

\author{
HUA CHEN $^{1^{*}}$, XINRAN JI $^{1^{*}}$, FEI SHE $^{2 *}$, YUAN GAO $^{1}$ and PEIFU TANG ${ }^{1}$ \\ ${ }^{1}$ Department of Orthopaedic Surgery, The General Hospital of People's Liberation Army (301 Hospital), Wukesong, \\ Beijing 100853; ${ }^{2}$ Department of Orthopaedic Surgery (304 Hospital), Haidian, Beijing 100048, P.R. China
}

Received January 20, 2016; Accepted October 6, 2016

DOI: 10.3892/ijmm.2016.2839

\begin{abstract}
Atrophic non-union is a serious complication of fractures. The underlying biological mechanisms involved in its pathogenesis are not yet completely understood. MicroRNAs (miRNAs or miRs) are a type of endogenous small non-coding RNA, which participate in various physiological and pathophysiological processes. In this study, differentially expressed miRNAs were screened in patients with atrophic nonunion. In total, 4 miRNAs (miR-149*, miR-221, miR-628-3p and miR-654-5p) were upregulated and 7 miRNAs (let- $7 \mathrm{~b}^{*}$, miR-220b, miR-513a-3p, miR-551a, miR-576-5p, miR-1236 and kshv-miR-K12-6-5p) were downregulated at the fracture sites in patients with atrophic non-union. Among the upregulated miRNAs, miR-628-3p and miR-654-5p expression was found to be persistently decreased during osteoblast differentiation, indicating their possible inhibitory effect on osteogenesis. Gain-of-function experiment demonstrated that miR-628-3p, but not miR-654-5p, attenuated osteoblast differentiation. Further, in silico analysis revealed that runt-related transcription factor 2 (RUNX2), the master transcript factor for osteoblast differentiation, was the target of miR-628-3p, which had two binding site-condense regions in the $3^{\prime}$ untranslated region. The exact binding site of miR-628-3p was further identified with luciferase reporter assay. In addition, the overexpression of miR-628-3p appeared to be associated with the suppression of RUNX2 expression at both the mRNA and protein level, suggesting that miR-628-3p inhibits osteoblast differentiation via RUNX2. On the whole, the findings of this study provide
\end{abstract}

Correspondence to: Dr Peifu Tang, Department of Orthopaedic Surgery, The General Hospital of People's Liberation Army (301 Hospital), 28 Fuxing Road, Wukesong, Beijing 100000, P.R. China

E-mail: tangpeifu301@126.com

${ }^{*}$ Co-first authorship

Abbreviations: GADPH, glyceraldehyde 3-phosphate dehydrogenase; RUNX2, runt-related transcription factor 2

Key words: miR-628-3p, osteoblast differentiation, runt-related transcription factor 2, non-union evidence of the upregulation of miR-628-3p in patients with atrophic non-union and that miR-628-3p may exert an inhibitory effect on osteogenesis via the suppression of its target gene, RUNX2. The study provides valuable insight into the pathogenesis of atrophic non-union and suggests new potential therapeutic targets for the treatment of this disorder.

\section{Introduction}

According to the US Food and Drug Administration, nonunion is established when a minimum of 9 months have elapsed since injury, and no signs of progression of fracture healing are demonstrable for 3 consecutive months. Non-union is also defined as the failure of a broken bone to heal at 6 months following fracture. The diagnosis of non-union is made upon the radiographic finding of a persistent radiolucent gap at the fracture site. Approximately 5-10\% of all fractures are complicated by delayed union or non-union (1). Despite the substantial progress made in the understanding of the mechanisms involved in fracture healing (2), non-union continues to be a challenge for orthopedic surgeons. In particular, atrophic non-union can be recalcitrant, resulting not only in chronic pain, but also in severe functional impairment (3).

The cause of atrophic non-union appears to be multifactorial and is the subject of intensive ongoing research. The factors associated with the development of non-union, include local factors, such as excessive mobility at the fracture site, fracture displacement, an impaired blood supply, open fractures, soft tissue interposition and infection, we well as systemic factors, such as osteoporosis, malnourishment, genetic predisposition, the administration of pharmacological agents (e.g., non-steroidal anti-inflammatory drugs), smoking and alcohol consumption (4).

Fracture healing is a dynamic physiological process involving a complex interplay of cells, mediators and growth factors. The healing process involves cellular recruitment, proliferation and differentiation under the guidance of signaling molecules, and the deposition of extracellular matrix components, that serve as a foundation for a successful bone healing response. The underling cellular and molecular mechanisms of this process are complex and are not yet completely understood. The disruption of any of the involved processes may lead to an impaired healing response, manifesting as delayed healing or non-union. The unraveling of the molecular mechanisms 
responsible for non-union fractures will pave the way for the development of novel therapeutic modalities.

MicroRNAs (miRNAs or miRs) are small non-coding RNA molecules that consists are 21-25 nucleotides in length and are typically excised from 60-110 nucleotide foldback RNA precursors. These molecules negatively regulate gene expression at the post-transcriptional level in a sequence-specific manner either by mRNA decay (predominant mechanism in plants) or by translational repression (predominant mechanism throughout the animal kingdom (5). Diverse expression patterns of miRNAs and altered miRNA expression under certain physiological conditions appear to indicate a range of hitherto unknown functions. Over the past two decades, research has indicated that miRNAs play a critical role in various physiological processes, such as development, cell proliferation, differentiation and apoptosis, as well as in many pathophysiological processes, such as inflammation and oncogenesis (6). Approximately 2,000 human miRNAs have been identified which may target approximately $60 \%$ of total human mRNAs; however, only a fraction of the identified miRNAs have been studied thus far (7-9).

Previous studies have documented that numerous miRNAs are associated with orthopedic disorders. Many miRNA, such as the miR-34 family, miR-143, miR-145 and miR-200b/c are associated with osteosarcoma, whereas the ones associated with osteosarcoma progression and metastases include miR-20a, miR-181c, miR-27a, miR-183, miR-93, miRNA-33b and miRNA-26a (10-17). Some miRNAs have been identified to have prognostic significance. For example, miR-451 is an unfavorable prognostic factor for both overall and disease-free survival in osteosarcoma (18). Low serum levels of miRNA-133b and miRNA-206 are predictors of a poor prognosis, whereas the combined elevation of miRNA-196a and miRNA-196b is predictor of unfavorable prognosis in patients with osteosarcoma $(19,20)$. Apart from osteosarcoma, miRNAs have also been associated with other orthopedic disorders. miRNA-210 has been shown to be involved in the regulation of post-menopausal osteoporosis (21). The expression of mature miR-17-5p has been shown to be significantly lower in non-traumatic osteonecrosis samples (22). Studies have also indicated that several miRNAs are differentially expressed in patients with intervertebral disc degeneration (23), and are thought to participate in the pathological process. For instance, miRNA-10b has been shown to promote nucleus pulposus cell proliferation by targeting HOXD10 in intervertebral disc degeneration (24). In addition, the knockdown of miR-140 has been shown to be associated with osteoarthritis-like changes, while miR-140 transgenic mice seem to develop resistance to joint damage due to osteoarthritis (25). Furthermore, miR-124 and miR-146a have been shown to be associated with rheumatoid arthritis; the administration of miR-146a has been shown to protect the joints from inflammatory damage in arthritic mice (26). Although the association between miRNAs and various other orthopedic disorders has also been investigated, the relevance of miRNAs in the pathogenesis of atrophic non-union remains to be elucidated.

In this study, specimens from patients with atrophic nonunion or bone healing following fracture were obtained for microarray analysis to characterize the differential miRNA expression profiles. The differentially expressed miRNAs were further verified by quantitative-polymerase chain reaction (qPCR) and these miRNA expression patterns during osteogenesis were also explored. miR-628-3p expression was high at the fracture site in patients with atrophic non-union, while it was decreased during osteoblast differentiation. Gain- of-function analysis revealed that miR-628-3p inhibited the differentiation of MG63 osteoblasts. In addition, in silico analysis and molecular analyses revealed that runt-related transcription factor 2 (RUNX2), the master gene of osteoblast differentiation, was the target gene of miR-628-3p, which strongly suggested the role of miR-628-3p/RUNX2 in atrophic non-union.

\section{Materials and methods}

Screening for differentially expressed miRNAs in patients with atrophic non-union. After obtaining approval from the Medical Ethics Committee of the General Hospital of People's Liberation Army (no. 301 Hospital of People's Liberation Army), 3 samples from patients with atrophic non-union and 3 samples from patients with normal fracture healing were collected and were stored at $-80^{\circ} \mathrm{C}$ until further testing. The samples were taken from the scar tissues of atrophic non-union and from the bony callus formed around the steel plate from fracture patients after healing. Written informed consent was obtained from each of the patients enrolled in this study.

RNA was extracted using TRIzol reagent (Life Technologies, Carlsbad, CA, USA) as per the manufacturers' instructions. The samples were grinded to a fine powder under liquid nitrogen. The RNA quality was assessed using a Bioanalyzer 2100 (Agilent Technologies, Inc., Santa Clara, CA, USA). miRNA expression profiling was carried out by KangChen Bio-tech (Shanghai, China) utilizing the Sanger miRBase 11.0 which contains 1,700 probes.

Cell culture, induction of differentiation and transfection. Human osteoblastic MG63 cells (American Type Culture Collection, Rockville, MD, USA) were routinely maintained in Dulbecco's modified Eagle's medium (DMEM) high glucose (Life Technologies) supplemented with $10 \%$ heat-inactivated fetal bovine serum (FBS; HyClone, Logan, UT, USA) at $37^{\circ} \mathrm{C}$ in a humidified atmosphere of $5 \% \mathrm{CO}_{2}$. The culture medium was changed every other day. For differentiation, the culture medium was replaced by differentiation medium containing bisphosphonates (alendronate $50 \mu \mathrm{M}$, pamidronate $50 \mu \mathrm{M}$ and zoledronate $50 \mathrm{mM}$ ) for 7 days, as described elsewhere (27). Cells cultured in a normal medium were used as a control. Cell transfection was performed using Lipofectamine 2000 (Life Technologies) as per the manufacturer's instructions. The cells were seeded in 6-well plates and cultured to 70-80\% confluence in antibiotic-free fresh medium. The cells were washed twice with DMEM prior to transfection. Subsequently, a mixture of Lipofectamine 2000 (Life Technologies) and miR-628-3p miRNA mimics or miR-654-5p mimics or miRNA mimic negative controls (Genepharma, Shanghai, China) were added followed by incubation for $6 \mathrm{~h}$. miRNA mimics negative control were used as a negative control (NC).

Detection of alkaline phosphatase (ALP) activity. ALP activity was measured using the alkaline phosphatase assay kit (Beyotime, Hangzhou, China) as per the manufacturer's instructions. 
Total protein was extracted using radioimmuno-precipitation assay (RIPA) lysis buffer. Following centrifugation of the cell lysate at $14,000 \mathrm{x} \mathrm{g}$ for $5 \mathrm{~min}$ at $4^{\circ} \mathrm{C}$, the total protein concentration in the lysate was determined by bicinchoninic acid assay. Equal volumes of substrate and cell lysate were added to each well of the 96-well plate and incubated for $10 \mathrm{~min}$. Following the addition of stop solution, ALP activity was evaluated by measuring the absorbance of the mixture with a test wavelength of $405 \mathrm{~nm}$ using an automicroplate reader (Infinite M200; Tecan, Grödig, Austria).

Alizarin Red $S$ staining. The cells in the 6-well plate were washed with phosphate-buffered saline (PBS) and fixed with 4\% paraformaldehyde. After washing with PBS 3 times, Alizarin Red staining solution (Sigma-Aldrich, St. Louis, MO, USA) was added followed by incubation for $30 \mathrm{~min}$. The cells was washed for another 3 times with PBS, and images were recorded using a Nikon CoolPix 950 digital camera (Nikon Instruments Inc., Shanghai, China).

3' Untranslated region (3'UTR) luciferase reporter assay. The 3'UTR luciferase reporter constructs of RUNX2 were made by cloning the 3'UTR region of the RUNX2 mRNA into the pGL3promoter vector (Promega, Madison, WI, USA). The primers for RUNX23'UTR-1 were 5'-acgtctagaagcatgaaatgctggagtga-3'(sense) and 5'-gatcatatgctgggtggcctacaaaggt-3' (antisense); for RUNX2 3'UTR-2 were 5'-acgtctagatggtccttctcaaacccacctt-3' (sense) and 5'-gatcatatgttcgttttctaaaaacaaca-3' (antisense); for RUNX2 3'UTR-1-mut were 5'-caaggggctgtggagtttggtgtcctagcttgtgtatgaattt gagctaga-3' (sense) and 5'-tctagctcaaattcatacacaagctaggaca ccaaactccacagcccc-3' (antisense); and for RUNX2 3'UTR-2-mut were 5'-gtcttactactactgtggaaccatgctagcattcctgggaattaaaat-3' and 5'-accacgctattttaattcccaggaatgctagcatggttccacagtagtagtaagac-3'. All the constructs were confirmed by sequencing analysis. 293 cells (ATCC, Manassas, VA, USA) were co-transfected with the luciferase reporter plasmid and the miR-628-3p mimics. After $24 \mathrm{~h}$, the luciferase activities were measured using the DualLuciferase reporter assay system (Promega) according to the manufacturer's instructions.

RNA quantification. Total RNA was extracted from the tissues or cultured cells using TRIzol reagent (Life Technologies) as per the manufacturer's instructions. A total of $1.5 \mu \mathrm{g}$ of RNA was used for reverse transcription. qPCR was performed using an ABI 7500 real-time PCR system and was executed using the SYBR-Green PCR master mix (both from Applied Biosystems, Foster City, CA, USA) with $2 \mu \mathrm{l}$ cDNA template in a $40 \mu \mathrm{l}$ final reaction mixture $\left(95^{\circ} \mathrm{C}\right.$ for $15 \mathrm{~min} ; 95^{\circ} \mathrm{C}$ for $15 \mathrm{sec}, 56^{\circ} \mathrm{C}$ for $30 \mathrm{sec}, 68^{\circ} \mathrm{C}$ for $\left.30 \mathrm{sec}, 40 \mathrm{cycles}\right)$. The average threshold cycle $(\mathrm{Ct})$ for each gene was determined from triplicate reactions; the relative expression level of mRNA or miRNAs was normalized to that of the internal controls, glyceraldehyde 3-phosphate dehydrogenase (GAPDH) or U6, using the $2^{-\Delta \Delta C t}$ method. The primers for ALP were 5'-ctaactccttagt gccagag-3' (sense) and 5'-catgatgacattcttagccac-3' (antisense); for collagen, type I, $\alpha 1$ (COL1A1) were 5'-gtgctaaaggtgcc aatggt-3' (sense) and 5'-ctcctcgctttccttcctct-3' (antisense); for osteocalcin (OC) were 5'-ctcacactcctcgccctattg-3' (sense) and 5'-cttggacacaaaggctgcac-3' (antisense); for GAPDH were 5'-tcagtggtggacctgacctg-3' (sense) and 5'-tgctgtagccaaattcgttg-3' (antisense); for miR-149* were 5'-agggagggacgggggctgtgc-3' (sense) and 5'-gcgagcacagaattaatacgac-3' (antisense); for miR-221 were 5'-agctacattgtctgctgggtttc-3' (sense) and 5'-gcgagcacagaa ttaatacgac-3' (antisense); for miR-628-3p were 5'-tctagtaagagt ggcagtcga-3' (sense) and 5'-gcgagcacagaattaatacgac-3' (antisense); for miR-654-5p were 5'-tggtgggccgcagaacatgtgc-3' (sense) and 5'-gcgagcacagaattaatacgac-3' (antisense); and for U6 were 5'-cgcttcggcagcacatatacta-3' (sense) and 5'-cgcttcacgaatttg cgtgtca-3' (antisense).

Western blot analysis. The cells were harvested and lysed with RIPA lysis buffer. Equal amounts of protein were loaded and separated via $10 \%$ sodium dodecyl sulfate-polyacrylamide gel electrophoresis (SDS-PAGE). Separated proteins were transferred onto $0.4-\mu \mathrm{m}$ polyvinylidene difluoride (PVDF) membranes. After blocking with 5\% skim milk for $3 \mathrm{~h}$, the PVDF membranes were incubated overnight with primary antibody against RUNX (H00000860-M06) or GAPDH (H00002597-M01) (1:1,000; Abnova, Taipei, China) diluted in blocking solution. Subsequently, the membranes were washed 3 times with Tris-buffered saline (TBS) with Tween-20 (TBST) [50 mM Tris- $\mathrm{HCl}(\mathrm{pH} 7.5), 150 \mathrm{mM} \mathrm{NaCl}$, and $0.05 \%$ Tween-20] and horseradish peroxidase-conjugated secondary antibodies (ZB-2305; ZSGB-BIO, Beijing, China) were incubated with the membranes for $1 \mathrm{~h}$ at room temperature. The membranes were washed twice with TBST and once with TBS, and soaked in enhanced chemiluminescence (ECL) reagent. Protein bands were visualized using the Western Blotting Systems ECL kit (Amersham, Piscataway, NJ, USA). Data obtained from western blot analysis were analyzed using Bio-Rad Quantity One 1-D Analysis software (Bio-Rad, Hercules, CA, USA).

Screening for target genes. we used the online software program, microRNA.org, to identify the candidate target genes of miR-628-3p. This was carried out by typing the name of microRNAs in the search bar.

Statistical analysis. All data are expressed as the means \pm standard deviation (SD). Statistical analyses were performed with SPSS (version 15.0; SPSS Inc., Chicago, IL, USA). The normality of distribution of all variables was verified before conducting the data analyses. Upon performing the Kolmogorov-Smirnov test, no significant differences were detected in the variance between the groups ( $F$ test). A one-way ANOVA was performed to detect statistically significant differences between more than 2 groups. Otherwise, a two-tailed Student's t-test was used to evaluate the differences between 2 groups. A P-value of $<0.05$ was considered to indicate a statistically significant difference.

\section{Results}

Screening for differentially expressed miRNAs in patients with atrophic non-union. Scar tissue from the 3 patients with atrophic non-union, and tissues from the bony callus from 3 patients with healed fractures were collected. The differentially expressed miRNAs were screened with miRNA microarray. A total of 4 miRNAs (miR-149*, miR-221, miR-628-3p and miR-654-5p) were was found to be significantly upregulated, whereas 7 miRNAs (let-7b*, miR-220b, 

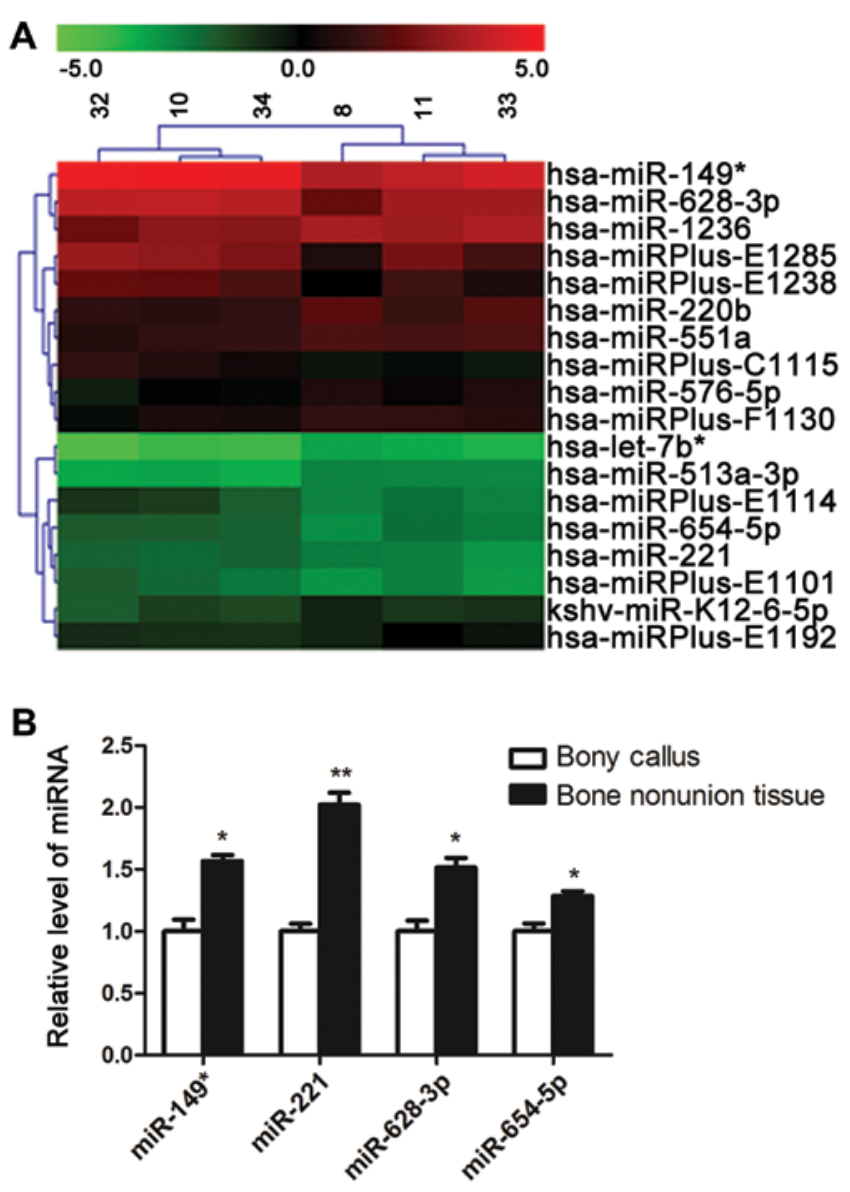

Figure 1. Screening for differentially expressed miRNAs between patients with atrophic non-union and patients with normally healing fracture. (A) Heatmap of the differentially expressed miRNAs in the scar tissue or the bony callus between patients with bone fracture non-union and patients with healed bone fractures. miRNAs were detected by microarray. The heatmap was generated from the average of normalized log-transformed fluorescent intensity for each data set. (B) The upregulated miRNAs in patients with atrophic non-union were verified by qPCR. Relative quantification was performed using the $2^{-\Delta \Delta C t}$ method with bony callus sample as a calibrator. Total RNA was poly-A tailed, reverse transcripted and then $\mathrm{qPCR}$ was performed. $\triangle \mathrm{CT}$ values obtained from qPCR were subjected to a paired t-test $(\triangle \mathrm{CT}=\mathrm{CT}$ miR - CT U6). The expression levels of miR-149*, miR-221, miR-628-3p and miR-654-5p at the site of bone non-union was significantly higher than those of bony callus tissues. ${ }^{*} \mathrm{P}<0.05$ and ${ }^{* *} \mathrm{P}<0.01$ compared with controls.

miR-513a-3p, miR-551a, miR-576-5p, miR-1236 and kshvmiR-K12-6-5p) were found to be downregulated in the patients with atrophic non-union (Fig. 1A).

The upregulation of miRNAs in the patients with atrophic non-union was verified by qPCR, which reconfirmed that the expression of the 4 miRNAs (miR-149*, miR-221, miR-628-3p and miR-654-5p) was increased in the scar tissues of patients with atrophic non-union (Fig. 1B).

Expression patterns of miRNAs upregulated in patients with atrophic non-union during osteoblast differentiation. To assess the association between the 4 upregulated miRNAs and osteoblast differentiation, we detected alterations in the expression levels of these miRNAs during the differentiation of MG63 osteoblasts. As shown in Fig. 2, miR-149* expression was consistent during the differentiation of MG63 osteo- blasts; miR-221 expression was increased at the beginning of differentiation (on day 3), but was decreased at the end of differentiation (on day 7); the expression of both miR-628-3p and miR-654-5p was found to be persistently downregulated during the differentiation process. These results suggest that miR-628-3p and miR-654-5p may have an inhibitory regulatory function in osteoblast differentiation.

miR-628-3p, but not miR-654-5p, attenuates osteoblast differentiation. To clarify the exact roles of miR-628-3p and miR-654-5p in the differentiation of osteoblasts, both miR-628-3p and miR-654-5p mimics were introduced into the MG63 cells and osteoblast differentiation was assessed. The expression levels of osteoblastic-related genes, including ALP, COL1A1 and OC were significantly upregulated following culture in osteoblastic differentiation medium (Fig. 3A). Under the differentiation-inducing conditions, transfection with miR-628-3p mimics markedly attenuated the increased expression of ossification-related genes, whereas the miR-654-5p mimics had no effect on the expression of these marker genes, compared with the group transfected with miRNA mimics negative control (induction $+\mathrm{NC}$ ). Further results revealed that ALP activity in the differentiated MG63 cells was inhibited by transfection with miR-628-3p mimics, but not by miR-654-5p 654-5p mimics (Fig. 3B). The results of in vitro cell mineral characteristics, as assessed by Alizarin Red S staining, were in line with the results mentioned above; namely transfection with miR-628-3p, but not miR-654-5p, mimics diminished MG63 mineralization induced by osteoblastic differentiation (Fig. 3C).

$R U N X 2$ is a target gene of miR-628-3p. Osteogenesis is a complex biological process involving multiple transcription factors. To identify the target gene by which miR-628-3p abates osteoblast differentiation potential, we searched the candidate target genes of miR-628-3p using the miRNA gene prediction software, microRNA.org. The master transcription factor of osteoblast differentiation, RUNX2, was predicted as a target gene of miR-628-3p. Two pairing regions were identified in the RUNX2 3' non-coding region, named RUNX2-3'UTR-1 and RUNX2-3'UTR-2 (Fig. 4A). To verify whether miR-628-3p can bind to these regions, luciferase reportervectors (pGL3-RUNX23'UTR-1 and pGL3-RUNX2-3'UTR-2) were constructed. In addition, the control vector were also generated with the mutated binding region (pGL3-RUNX2-3'UTR-1-mut and pGL3-RUNX2-3'UTR-2-mut) (Fig. 4B). miR-628-3p mimics were transfected into 293 cells with either the reporter vector or control vector. The results revealed that luciferase activity specifically weakened in the cells transfected with miR-628-3p mimics and pGL3-RUNX2-3'UTR-1, but not in other group, indicating that miR-628-3p can specifically bind to RUNX23'UTR-1 and may play a role in the suppression of RUNX2 expression (Fig. 4C and D).

To verify this hypothesis, we examined the RUNX2 protein level following transfection with miR-628-3p mimics. The results exhibited a markedly decreased RUNX2 expression as compared with the control group. We further detected the effects of miR-628-3p on the RUNX2 mRNA levels, which demonstrated that miR-628-3p decreased the RUNX2 mRNA levels (Fig. 4E). 
miR-149*

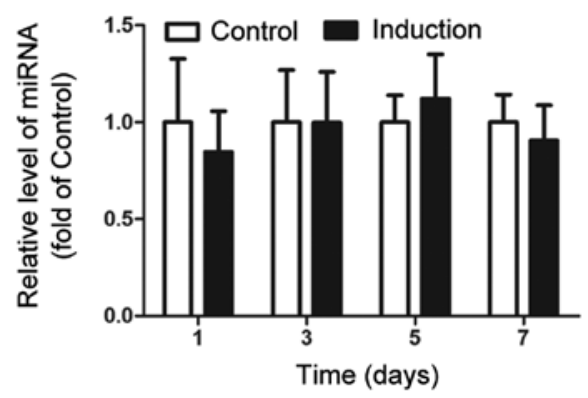

miR-628-3p

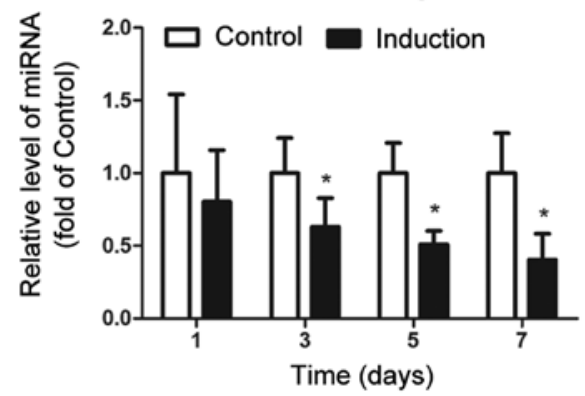

miR-221

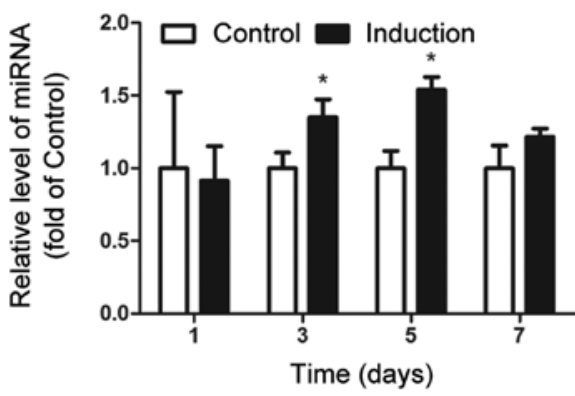

miR-654-5p

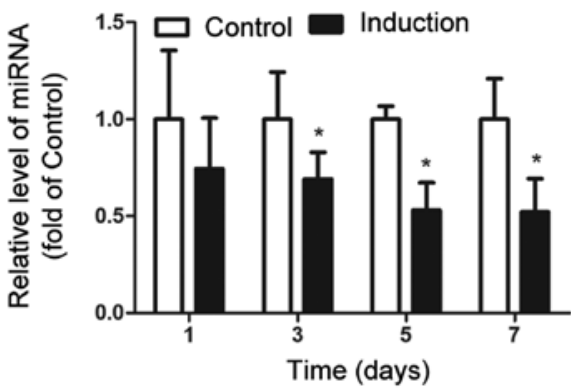

Figure 2. Expression pattern of atrophic non-union upregulated miRNAs during osteoblast differentiation. MG63 osteoblasts that were cultured in basal medium and osteogenic induction medium, respectively, for 1,3,5 and 7 days. The miRNA levels were assayed by qPCR and expressed as the fold of the control group. ${ }^{*} \mathrm{P}<0.05$ compared to the control group.

A

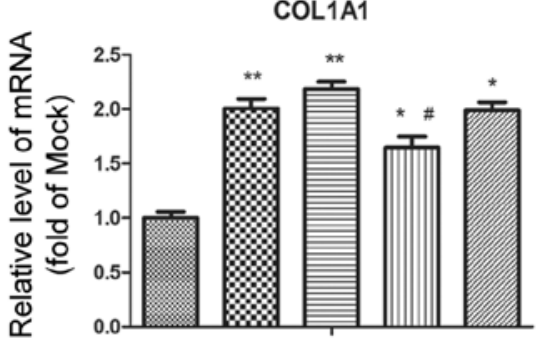

oc

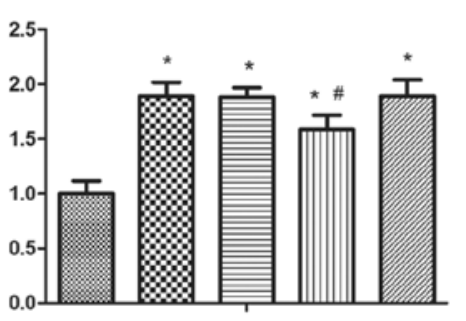

B

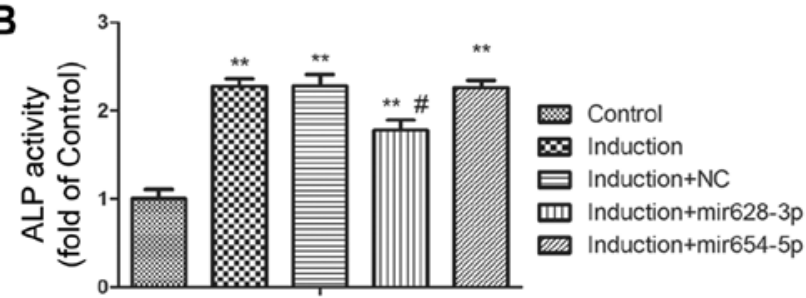

ALP

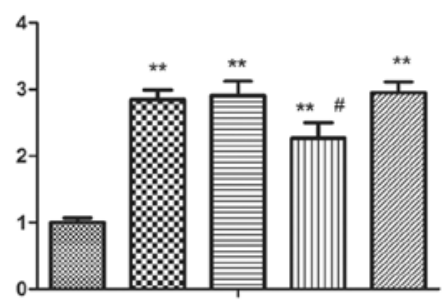

Control

Induction

Induction+NC

Induction+miR-628-3p

Induction+miR-654-5p

C

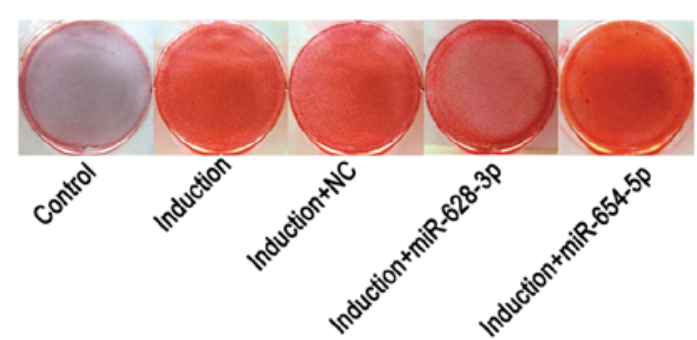

Figure 3. miR-628-3p, but not miR-654-5p, attenuates osteoblast differentiation. (A) Effect of miR-628-3p and miR-654-5p on the expression of osteogenesis differentiation marker genes during osteoblast differentiation. MG63 cells that were treated under different conditions (basal medium and osteogenic induction medium). There were other 3 groups which were transfected by NC (miRNA mimics negative control), miR-628-3p and miR-654-5p mimics before being cultured in osteogenic induction medium. The mRNA levels were assayed by qPCR and expressed as the fold of the control group. (B) Effects of miR-628-3p and miR-654-5p on the activity of alkaline phosphatase (APL) in the differentiated MG63 cells. The activity of APL was expressed as the fold of the control group. (C) Effects of miR-628-3p and miR-654-5p on the mineralization of the differentiated MG63 cells. Mineralized matrix of the MG63 cells was examined by Alizarin Red $\mathrm{S}$ staining. ${ }^{*} \mathrm{P}<0.05$ and ${ }^{* *} \mathrm{P}<0.01$ compared with the control group; ${ }^{*} \mathrm{P}<0.05$ compared with the NC group. 
A

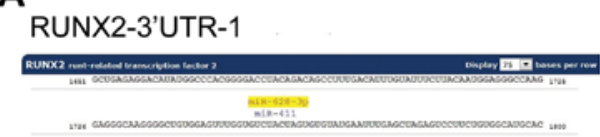

\section{RUNX2-3'UTR-2}

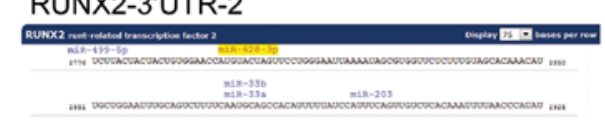

C

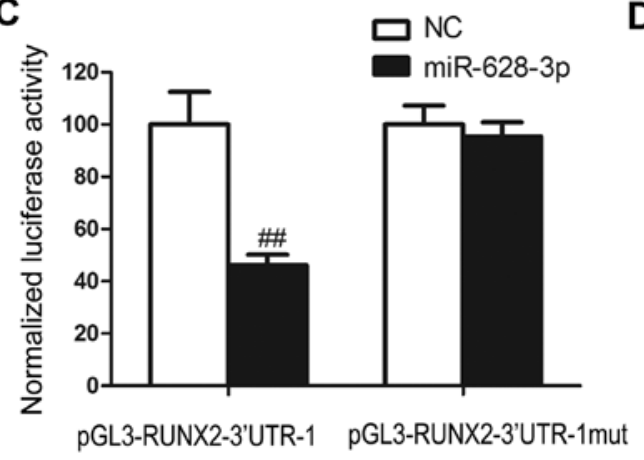

B

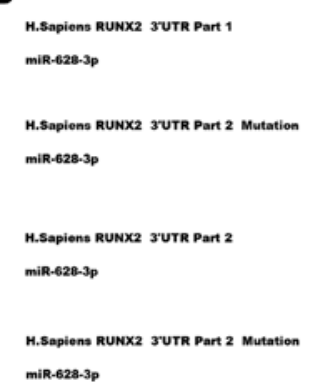

D

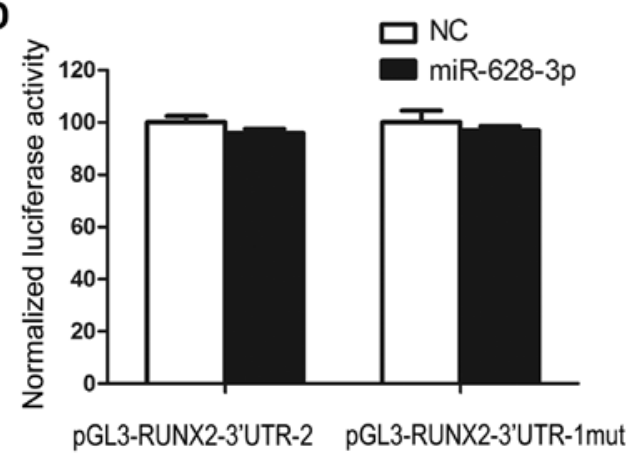

E
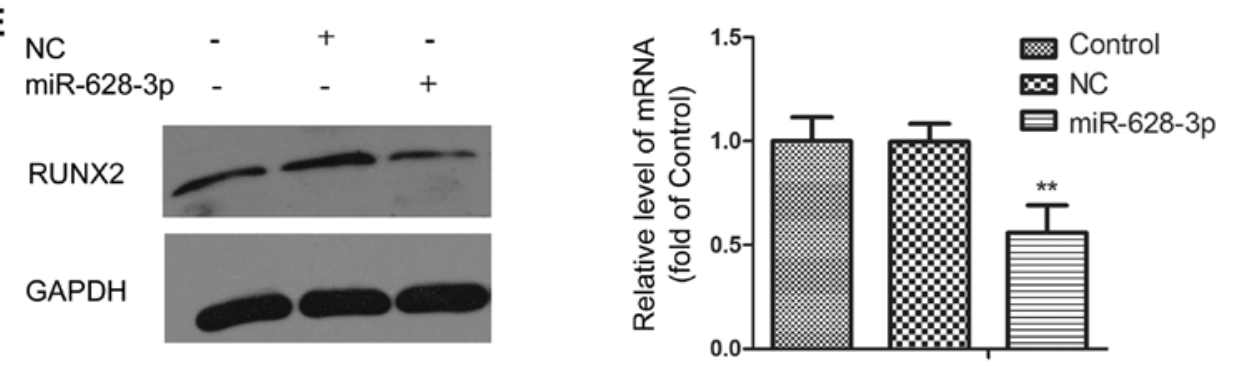

Figure 4. Runt-related transcription factor 2 (RUNX2) is a target gene for miR-628-3p. (A) Predicted duplex formation between miR-628-3p and the targeted RUNX2-3' untranslated region (3'UTR)-1 or RUNX2-3'UTR-2; (B) The sequences of RUNX2-3'UTR-1, RUNX2-3'UTR-2, RUNX2-3'UTR-1mut, and RUNX2-3'UTR-2mut. RUNX2-3'UTR-1mut and RUNX2-3'UTR-2mut sequences were identical to the wild-type sequences, except for a 6 bp substitution; (C and D) miR-628-3p targets the 3'UTR-1 (C), but not 3'UTR-2 (D), of RUNX2 mRNA. pGL3m-RUNX2-3'UTR reporter plasmids or mutation plasmids were co-transfected into 293 cells with NC or miR-628-3p mimics. Luciferase activity was normalized to Renilla luciferase expression. ${ }^{\#} \mathrm{P}<0.01$ compared with corresponding NC group. (E) Effect of miR-628-3p on RUNX2 expression at both protein and mRNA levels. RUNX2 protein was measured in MG63 cells by western blot analysis at $48 \mathrm{~h}$ following transfection with synthetic miR-628-3p mimics. Glyceraldehyde 3-phosphate dehydrogenase (GAPDH) was used as an internal loading control. ${ }^{* *} \mathrm{P}<0.01$ compared with the control group.

\section{Discussion}

In this study, we demonstrated the differential expression of miRNAs between patients with atrophic non-union and patients with bone healing following fracture through screening with miRNA microarray. A total of 4 upregulated and 7 downregulated miRNAs were identified. Amongst the upregulated miRNAs, miR-628-3p and miR-654-5p expression was decreased persistently during osteoblast differentiation. miR-628-3p mimics, but not miR-654-5p mimics, attenuated the osteogenic differentiation of MG63 cells. Furthermore, RUNX2 was predicted as a candidate target gene by in silico analysis. Further results verified that miR-628-3p decreased the RUNX2 level at both the protein and mRNA level.

Atrophic non-union is triggered by multiple causes. Impaired blood circulation is considered to be the most importance factor (28). However, with the progress in research on macrovascular techniques, this view has been challenged in recent years. Multiple studies have indicated that blood supply is not reduced in atrophic non-union and angiogenesis also seems unimpaired. In a study by Santavirta et al (29), pathology specimens from 10 patients with non-union did not reveal any evidence of decreased vascular density. Besides, in another study, the blood supply in atrophic non-union was reported as being more than adequate, when compared with hypertrophic non-union (30). Increasingly, researchers are accepting the opinion that insufficient osteogenesis plays a crucial role in non-union. Bone fracture healing involves hematoma formation and its filling in by a fibrin network; subsequently, cells grow along a fibrin meshwork to from procallus and fibrocartilaginous callus, followed by the deposition of calcium salt that converts the fibrocartilaginous callus into the spongy bone that is characteristic of the bony callus. In the final step, the bony callus remodels into a normal shape. During this process, impaired osteogenic capability results in atrophic non-union. Many factors and/or signaling pathways involved in osteogenesis seem to improve bone healing (30). The transplantation of pluripotent stem cells, such as mesenchymal stem cells, which 
can undergo osteogenic differentiation, has an obvious therapeutic benefit in patients with non-union (31). As shown in this study, miR-628-3p is highly expressed in atrophic non-union and can inhibit osteogenesis, indicating its negative role in atrophic non-union modulated via the suppression of osteogenesis.

miRNAs control osteoblastogenesis. A general null mutation of Dicer has been shown to prevent the formation of a normal vertebrate body plan at gastrulation, suggesting a critical role of miRNAs in the formation of bone. Furthermore, the knockdown of Dicer and Drosha using lentiviruses expressing shRNAs, has been shown to inhibit the osteogenic differentiation of human multipotent stromal cells (32). Multiple miRNAs regulate osteoblast differentiation, with some of them exerting an inhibitory effect. For instance, miR-34s inhibit osteoblast proliferation and differentiation in mice by targeting SATB homeobox 2 (SATB2) (33). Furthermore, miRNA-100 regulates the osteogenic differentiation of human adipose-derived mesenchymal stem cells by targeting bone morphogenetic protein receptor type II (BMPR2) (34). miR-214 targets activating transcription factor 4 (ATF4) to inhibit bone formation (35). The miR-93/Sp7 function loop mediates osteoblast mineralization in osteogenesis (36). Other microRNAs have been shown to have a strengthening function. For instance, the upregulation of miR-22 promotes osteogenic differentiation by repressing histone deacetylase (HDAC)6 protein expression in human adipose tissue-derived mesenchymal stem cells (37), and the overexpression of miR-2861 promotes osteoblast differentiation by suppressing HDAC5 in primary osteoblasts (38). In this study, miR-628-3p was a found to be involved in osteoblast differentiation.

Until now, studies investigating the function of miR-628-3p, have been exceedingly rare, with only a few studies investigating a possible association between this miRNA and human diseases. Cui et al reported a marked alteration of serum miR-628-3p levels in patients with hand-foot-and-mouth disease, suggesting its potential value as a candidate molecular marker to differentiate patients with enterovirus infections from healthy individuals (39). Another recent study highlighted the potential of this miRNA for helping distinguish sera of patients with pancreatic cancer from those of healthy persons (40). Furthermore, another study indicated that miR-628-3p in platelets may influence mRNA processing in patients with diabetes mellitus (41). In the present study, we demonstrated that miR-628-3p expression was increased in the scar tissue of patients with atrophic non-union, and that its expression was downregulated during osteoblast differentiation. Further experiments proved that this miRNA suppresses osteoblast differentiation. To the best of our knowledge, this is the first study to profile the functional aspects of miR-628-3p.

The master transcription factors, such as RUNX2 and SP7-Osterix, have autonomous effects on osteoblast differentiation. The protein coding sequence of RUNX2 is approximtely 1,500 nucleotides, whereas, its 3'UTR is much longer (of the order of 3,000 nucleotides), which make this gene the common targeting gene for miRNAs regulating osteogenesis. With the established 3'UTR sequence of RUNX2, Zhang et al identified $>11$ miRNAs binding to the 3 'UTR of RUNX2, i.e., miR-23a, miR-30c, miR-34c, miR-133a, miR-135a, miR-137, miR-204, miR-205, miR-217, miR-218 and miR-338. All these miRNA differ in their expression level in osteogenic and non-osteogenic cells, and exhibit a regulatory function in osteoblast differentiation (42). Some of these miRNAs were also identified in parallel studies (43-46). Apart from these 11 miRNAs, it has also been demonstrated that RUNX2 is also controlled by other miRNAs. For example, miR-204 has been shown to regulate RUNX2 protein levels in mesenchymal progenitor cells, while miRNA-103a acts as a mechano-sensitive miRNA suppressing Runx 2 to inhibit bone formation (47). This study demonstrated that the binding of miR-628-3p to the RUNX2 3'UTR region suppressed RUNX2 expression, thus adding a new member to the growing list of miRNAs that regulate RUNX2 expression.

A major limitation of this study was the fact that we did not verify the function of miR-628-3p in vivo, and, whether this miRNA has a potential therapeutic role in atrophic non-union. Whether the overexpression of RUNX2 can reverse the inhibitory effects of miR-628-3p on osteoblast differentiation also needs to be verified. In addition, the functions of the miRNAs found to be downregulated in atrophic non-union also need to be assessed further.

In conclusion, to the best of our knowledge, the present study is the first to elucidate the role of miRNAs in the pathological process of atrophic non-union. We identified an miRNA i.e., miR-628-3p, which was upregulated in patients with atrophic non-union and exerted an inhibitory effect on osteogenesis through the suppression of its target gene, RUNX2. The findings of our study may provide insight into the development of novel therapeutic targets for the management of atrophic non-union.

\section{Acknowledgements}

This study was supported by grants from the National Natural Science Foundation of China (no. 81000803 to H.C.).

\section{References}

1. Tzioupis C and Giannoudis PV: Prevalence of long-bone nonunions. Injury 38 (Suppl 2): S3-S9, 2007.

2. Einhorn TA and Gerstenfeld LC: Fracture healing: mechanisms and interventions. Nat Rev Rheumatol 11: 45-54, 2015.

3. Dimitriou R, Kanakaris N, Soucacos PN and Giannoudis PV: Genetic predisposition to non-union: evidence today. Injury 44 (Suppl 1): S50-S53, 2013.

4. Copuroglu C, Calori GM and Giannoudis PV: Fracture non-union: Who is at risk? Injury 44: 1379-1382, 2013.

5. He L and Hannon GJ: MicroRNAs: Small RNAs with a big role in gene regulation. Nat Rev Genet 5: 522-531, 2004.

6. Calin GA and Croce CM: MicroRNA signatures in human cancers. Nat Rev Cancer 6: 857-866, 2006.

7. Lim LP, Glasner ME, Yekta S, Burge CB and Bartel DP: Vertebrate microRNA genes. Science 299: 1540, 2003.

8. Kozomara A and Griffiths-Jones S: miRBase: Integrating microRNA annotation and deep-sequencing data. Nucleic Acids Res 39: D152-D157, 2011.

9. Friedländer MR, Lizano E, Houben AJ, Bezdan D, Báñez-Coronel M, Kudla G, Mateu-Huertas E, Kagerbauer B, González J, Chen KC, et al: Evidence for the biogenesis of more than 1,000 novel human microRNAs. Genome Biol 15: R57, 2014.

10. Xu N, Li Z, Yu Z, Yan F, Liu Y, Lu X and Yang W: MicroRNA-33b suppresses migration and invasion by targeting c-Myc in osteosarcoma cells. PLoS One 9: e115300, 2014.

11. He C, Xiong J, Xu X, Lu W, Liu L, Xiao D and Wang D: Functional elucidation of MiR-34 in osteosarcoma cells and primary tumor samples. Biochem Biophys Res Commun 388: 35-40, 2009.

12. Zhuo W, Ge W, Meng G, Jia S, Zhou X and Liu J: MicroRNA-20a promotes the proliferation and cell cycle of human osteosarcoma cells by suppressing early growth response 2 expression. Mol Med Rep 12: 4989-4994, 2015. 
13. Mori F, Sacconi A, Canu V, Ganci F, Novello M, Anelli V, Covello R, Ferraresi V, Muti P, Biagini R, et al: miR-181c associates with tumor relapse of high grade osteosarcoma. Oncotarget 6: 13946-13961, 2015

14. Salah Z, Arafeh R, Maximov V, Galasso M, Khawaled S, Abou-Sharieha S, Volinia S, Jones KB, Croce CM and Aqeilan RI: miR-27a and miR-27a* contribute to metastatic properties of osteosarcoma cells. Oncotarget 6: 4920-4935, 2015.

15. Mu Y, Zhang $\mathrm{H}$, Che $\mathrm{L}$ and $\mathrm{Li} \mathrm{K}$ : Clinical significance of microRNA-183/Ezrin axis in judging the prognosis of patients with osteosarcoma. Med Oncol 31: 821, 2014.

16. Kawano M, Tanaka K, Itonaga I, Ikeda S, Iwasaki $\mathrm{T}$ and Tsumura H: microRNA-93 promotes cell proliferation via targeting of PTEN in Osteosarcoma cells. J Exp Clin Cancer Res 34: 76, 2015.

17. Qu F, Li CB, Yuan BT, Qi W, Li HL, Shen XZ, Zhao G, Wang JT and Liu YJ: MicroRNA-26a induces osteosarcoma cell growth and metastasis via the $\mathrm{Wnt} / \beta$-catenin pathway. Oncol Lett 11 : 1592-1596, 2016

18. Yuan J, Lang J, Liu C, Zhou K, Chen L and Liu Y: The expression and function of miRNA-451 in osteosarcoma. Med Oncol 32: 324, 2015.

19. Zhang C, Yao C, Li H, Wang G and He X: Serum levels of microRNA-133b and microRNA-206 expression predict prognosis in patients with osteosarcoma. Int J Clin Exp Pathol 7: 4194-4203, 2014.

20. Zhang C, Yao C, Li H, Wang G and He X: Combined elevation of microRNA-196a and microRNA-196b in sera predicts unfavorable prognosis in patients with osteosarcomas. Int J Mol Sci 15: 6544-6555, 2014.

21. Liua XD, Caia F, Liu L, Zhang Y and Yang AL: microRNA-210 is involved in the regulation of postmenopausal osteoporosis through promotion of VEGF expression and osteoblast differentiation. Biol Chem 396: 339-347, 2015

22. Jia J, Feng X, Xu W, Yang S, Zhang Q, Liu X, Feng Y and Dai Z: miR-17-5p modulates osteoblastic differentiation and cell proliferation by targeting SMAD7 in non-traumatic osteonecrosis. Exp Mol Med 46: e107, 2014

23. Zhao B, Yu Q, Li H, Guo X and He X: Characterization of microRNA expression profiles in patients with intervertebral disc degeneration. Int J Mol Med 33: 43-50, 2014.

24. Yu X, Li Z, Shen J, Wu WK, Liang J, Weng X and Qiu G: MicroRNA-10b promotes nucleus pulposus cell proliferation through RhoC-Akt pathway by targeting HOXD10 in intervetebral disc degeneration. PLoS One 8: e83080, 2013.

25. Miyaki S, Sato T, Inoue A, Otsuki S, Ito Y, Yokoyama S, Kato Y, Takemoto F, Nakasa T, Yamashita S, et al: MicroRNA-140 plays dual roles in both cartilage development and homeostasis. Genes Dev 24: 1173-1185, 2010

26. Nakasa T, Shibuya $H$, Nagata $Y$, Niimoto $T$ and Ochi $M$ : The inhibitory effect of microRNA-146a expression on bone destruction in collagen-induced arthritis. Arthritis Rheum 63 $1582-1590,2011$

27. Lee YJ, Jeong JK, Seol JW, Xue M, Jackson C and Park SY: Activated protein $\mathrm{C}$ differentially regulates both viability and differentiation of osteoblasts mediated by bisphosphonates. Exp Mol Med 45: e9, 2013.

28. Brinker MR and Bailey DE Jr: Fracture healing in tibia fractures with an associated vascular injury. J Trauma 42: 11-19, 1997.

29. Santavirta S, Konttinen YT, Nordström D, Mäkelä A, Sorsa T, Hukkanen M and Rokkanen P: Immunologic studies of nonunited fractures. Acta Orthop Scand 63: 579-586, 1992

30. Moghaddam A, Elleser C, Biglari B, Wentzensen A and Zimmermann G: Clinical application of BMP 7 in long bone non-unions. Arch Orthop Trauma Surg 130: 71-76, 2010.

31. Fayaz HC, Giannoudis PV, Vrahas MS, Smith RM, Moran C, Pape HC, Krettek C and Jupiter JB: The role of stem cells in fracture healing and nonunion. Int Orthop 35: 1587-1597, 2011.

32. Oskowitz AZ, Lu J, Penfornis P, Ylostalo J, McBride J, Flemington EK, Prockop DJ and Pochampally R: Human multipotent stromal cells from bone marrow and microRNA: Regulation of differentiation and leukemia inhibitory factor expression. Proc Natl Acad Sci USA 105: 18372-18377, 2008.
33. Wei J, Shi Y, Zheng L, Zhou B, Inose H, Wang J, Guo XE, Grosschedl R and Karsenty G: miR-34s inhibit osteoblast proliferation and differentiation in the mouse by targeting SATB2. J Cell Biol 197: 509-521, 2012

34. Zeng Y, Qu X, Li H, Huang S, Wang S, Xu Q, Lin R, Han Q, Li J and Zhao RC: MicroRNA-100 regulates osteogenic differentiation of human adipose-derived mesenchymal stem cells by targeting BMPR2. FEBS Lett 586: 2375-2381, 2012.

35. Wang X, Guo B, Li Q, Peng J, Yang Z, Wang A, Li D, Hou Z, Lv K, Kan G, et al: miR-214 targets ATF4 to inhibit bone formation. Nat Med 19: 93-100, 2013

36. Yang L, Cheng P, Chen C, He HB, Xie GQ, Zhou HD, Xie $\mathrm{H}$, Wu XP and Luo XH: miR-93/Sp7 function loop mediates osteoblast mineralization. J Bone Miner Res 27: 1598-1606, 2012.

37. Huang S, Wang S, Bian C, Yang Z, Zhou H, Zeng Y, Li H, Han Q and Zhao RC: Upregulation of miR-22 promotes osteogenic differentiation and inhibits adipogenic differentiation of human adipose tissue-derived mesenchymal stem cells by repressing HDAC6 protein expression. Stem Cells Dev 21: 2531-2540, 2012

38. Li H, Xie H, Liu W, Hu R, Huang B, Tan YF, Xu K, Sheng ZF, Zhou HD, Wu XP and Luo XH: A novel microRNA targeting HDAC5 regulates osteoblast differentiation in mice and contributes to primary osteoporosis in humans. J Clin Invest 119: 3666-3677, 2009.

39. Cui L, Qi Y, Li H, Ge Y, Zhao K, Qi X, Guo X, Shi Z, Zhou M, Zhu B, et al: Serum microRNA expression profile distinguishes enterovirus 71 and coxsackievirus 16 infections in patients with hand-foot-and-mouth disease. PLoS One 6: e27071, 2011.

40. Li A, Yu J, Kim H, Wolfgang CL, Canto MI, Hruban RH and Goggins M: MicroRNA array analysis finds elevated serum miR-1290 accurately distinguishes patients with low-stage pancreatic cancer from healthy and disease controls. Clin Cancer Res 19: 3600-3610, 2013.

41. Stratz C, Nührenberg T, Fiebich BL, Amann M, Kumar A, Binder H, Hoffmann I, Valina C, Hochholzer W, Trenk D, et al: Controlled type II diabetes mellitus has no major influence on platelet micro-RNA expression. Results from micro-array profiling in a cohort of 60 patients. Thromb Haemost 111: 902-911, 2014.

42. Zhang Y, Xie RL, Croce CM, Stein JL, Lian JB, van Wijnen AJ and Stein GS: A program of microRNAs controls osteogenic lineage progression by targeting transcription factor Runx 2 . Proc Natl Acad Sci USA 108: 9863-9868, 2011.

43. Li Z, Hassan MQ, Volinia S, van Wijnen AJ, Stein JL, Croce CM, Lian JB and Stein GS: A microRNA signature for a BMP2-induced osteoblast lineage commitment program. Proc Natl Acad Sci USA 105: 13906-13911, 2008.

44. Li Z, Hassan MQ, Jafferji M, Aqeilan RI, Garzon R, Croce CM, van Wijnen AJ, Stein JL, Stein GS and Lian JB: Biological functions of miR-29b contribute to positive regulation of osteoblast differentiation. J Biol Chem 284: 15676-15684, 2009.

45. Hassan MQ, Gordon JA, Beloti MM, Croce CM, van Wijnen AJ, Stein JL, Stein GS and Lian JB: A network connecting Runx2, SATB2, and the miR-23a 27a 24-2 cluster regulates the osteoblast differentiation program. Proc Natl Acad Sci USA 107: 19879-19884, 2010.

46. Hassan MQ, Maeda Y, Taipaleenmaki H, Zhang W, Jafferji M, Gordon JA, Li Z, Croce CM, van Wijnen AJ, Stein JL, et al: miR-218 directs a Wnt signaling circuit to promote differentiation of osteoblasts and osteomimicry of metastatic cancer cells. J Biol Chem 287: 42084-42092, 2012.

47. Zuo B, Zhu J, Li J, Wang C, Zhao X, Cai G, Li Z, Peng J, Wang P, Shen C, et al: microRNA-103a functions as a mechno-sensitive microRNA to inhibit bone formation through targeting Runx2. J Bone Miner Res 30: 330-245, 2015. 